\title{
Riscos causados pelo uso indiscriminado de medicamentos para emagrecer
}

\author{
Risks caused by the indiscriminate use of slimming drugs \\ Riesgos provocados por el uso indiscriminado de medicamentos para adelgazar
}

Recebido: 06/08/2021 | Revisado: 10/08/2021 | Aceito: 16/08/2021 | Publicado: 17/08/2021

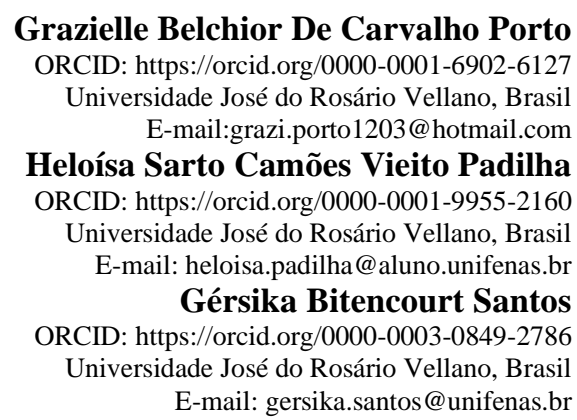

\begin{abstract}
Resumo
Objetivo: Descrever os riscos e efeitos adversos causados pelo uso indiscriminado dos medicamentos emagrecedores utilizados para tratamento do sobrepeso/obesidade. Métodos: Trata-se de uma revisão sistemática, baseada em estudos sobre a obesidade e o tratamento dela, com foco nos fatores adversos descritos nesses trabalhos. Essa busca foi feita em bases científicas, como SciELO, PubMed, Google Scholar, Bireme, Plataforma do CAPES e Lilacs. Resultados: Do total de artigos encontrados (80.600 artigos) os quais analisam variados fármacos utilizados para o tratamento da obesidade, foram selecionados 14 para compor esta revisão bibliográfica sistemática. Conclusão: há casos que se faz necessário o tratamento medicamentoso, e esse sendo bem orientado, prescrito e fiscalizado, os benefícios serão maiores que os prejuízos causados pelos efeitos adversos. Entretanto, na realidade, a maioria das vezes é uma utopia esse tratamento bem orientado e prescrito, assim esses efeitos adversos tomam maiores proporções sobrepondo os benefícios.
\end{abstract}

Palavras-chave: Obesidade; Riscos; Emagrecimento; Anorexígenos.

\begin{abstract}
Objective: To describe the risks and adverse effects caused by the indiscriminate use of slimming drugs used to treat overweight/obesity. Methods: This is a systematic review, based on studies on obesity and its treatment, focusing on the adverse factors described in these studies. This search was carried out on scientific bases such as SciELO, PubMed, Google Scholar, Bireme, CAPES Platform and Lilacs. Results: From the total of articles found (80,600 articles) which analyze different drugs used for the treatment of obesity, 14 were selected to compose this systematic literature review. Conclusion: there are cases in which drug treatment is necessary, and this being well oriented, prescribed and supervised, the benefits will be greater than the losses caused by the adverse effects. However, in reality, most of the times, this well-guided and prescribed treatment is a utopia, so these adverse effects take on greater proportions, outweighing the benefits.
\end{abstract}

Keywords: Obesity; Risks; Weight Loss; Anorexigenic.

\section{Resumen}

Objetivo: Describir los riesgos y efectos adversos provocados por el uso indiscriminado de fármacos adelgazantes utilizados para tratar el sobrepeso / obesidad. Métodos: Se trata de una revisión sistemática, basada en estudios sobre la obesidad y su tratamiento, centrada en los factores adversos descritos en estos estudios. Esta búsqueda se realizó sobre bases científicas como SciELO, PubMed, Google Scholar, Bireme, CAPES Platform y Lilacs. Resultados: Del total de artículos encontrados (80.600 artículos) que analizan diferentes fármacos utilizados para el tratamiento de la obesidad, se seleccionaron 14 para componer esta revisión bibliográfica sistemática. Conclusión: hay casos en los que es necesario el tratamiento farmacológico, y bien orientado, prescrito y supervisado, los beneficios serán mayores que las pérdidas ocasionadas por los efectos adversos. Sin embargo, en realidad, la mayoría de las veces este tratamiento bien orientado y prescrito es una utopía, por lo que estos efectos adversos adquieren mayores proporciones, superando los beneficios.

Palabras clave: Obesidad; Riesgos; Pérdida de peso;Anorexigénico. 


\section{Introdução}

A obesidade é definida pela Organização Mundial de Saúde (OMS), como um acúmulo anormal ou excessivo de gordura que acarreta prejuízos à saúde, e foi incluída, em 1948, na Classificação Internacional de Doenças. A obesidade é considerada uma doença por predispor o indivíduo à morte precoce e ao desenvolvimento de enfermidades, e por ser também um dos mais graves problemas de saúde. A principal forma de diagnóstico de obesidade é através do Índice de Massa Corporal (IMC). Segundo a OMS, um IMC que está acima de $30 \mathrm{~kg} / \mathrm{m}^{2}$, é indício de obesidade (ABESO, 2016; Organização Mundial Da Saúde, 2019).

O Instituto Brasileiro de Geografia e Estatística (IBGE), através da Pesquisa Nacional de Saúde de 2019, revela que o número de pessoas obesas maiores de vinte anos, entre os anos de 2003 e 2019, mais que dobrou, devido esse grande aumento, a obesidade pode ser considerada uma epidemia. Por isso, a obesidade deve ser tratada como qualquer outra doença, incluindo além de mudanças nos hábitos de vida, um acompanhamento psicológico e em alguns casos a introdução de terapia medicamentosa (Silva \& Cantisani, 2018; Paim \& Kovaleski, 2020; Tylka. et al., 2014; Ministério Da Saúde, 2020).

A sociedade não assimila a obesidade como qualquer outra doença existente, em que o doente merece uma atenção e cuidado especial. Pelo contrário, o obeso é visto como indivíduo incompetente, desleixado e sem controle, enquanto o corpo que se encaixa nos padrões é associado a disciplina e força de vontade. Um novo termo surgiu na modernidade, a gordofobia, usado para designar uma aversão, preconceito e uma opressão a indivíduos obesos, por não se enquadrarem no perfil idealizado pela sociedade atual (WU \& Berry, 2017; Lucena, 2020; Coutinho, 2017; Silva, \& Cantisani, 2018; Tarozo \& Pessa, 2020).

O estigma criado na sociedade contemporânea em relação às pessoas obesas, têm resultado em graves danos de ordem física e psicossocial. Assim, se tornou uma ferramenta para discriminar e excluir o indivíduo, além de colaborar no surgimento de problemas como bullying, depressão, suicídio, abuso de anorexígenos, cirurgias plásticas, distorção da própria imagem, entre outros. Isso tudo é reflexo de uma geração facilmente influenciável pela mídia, sem nenhum senso crítico (Reis \& Richter , 2014; Lucena, 2016; Tarozo \& Pessa, 2020; Rangeli, 2018; Lima et al., 2013).

No Brasil, o projeto $\mathrm{n}^{\circ} 2.431 / 11$ autoriza a produção, comercialização e consumo, sob prescrição médica, de medicamentos anorexígenos para o tratamento da obesidade. Para o Conselho Federal de Medicina (CFM) esse projeto é de fato uma conquista, visto que esses medicamentos são ferramentas essenciais para um tratamento eficiente da obesidade. Segundo a Sociedade Brasileira de Endocrinologia e Metabologia, (SBEM), "o uso racional desses medicamentos sempre foi defendido pela SBEM, e somos totalmente contrários ao uso desses medicamentos de maneira indiscriminada, como aconteceu no passado. Mas retirá-los do mercado nunca foi a solução para o problema”" (De Menezes, et al.; Silva, \& Cantisani, 2018).

Por outro lado, o que é preocupante é a banalização do uso de medicamentos emagrecedores. Muitas pessoas que não se enquadram no perfil do paciente obeso com necessidade de tratamento têm acesso a essas medicações e fazem o uso de forma incoerente, sem prescrição e orientação correta, sendo expostas a uma série de eventos colaterais e a dependência medicamentosa (Paim \& Kovaleski, 2020).

Nesse contexto, o objetivo do presente trabalho é avaliar, através de uma revisão bibliográfica os efeitos adversos dos medicamentos utilizados de maneira indiscriminada para emagrecer, assim como os riscos que eles trazem para a saúde.

\section{Metodologia}

Este estudo é uma revisão bibliográfica sistemática (Pereira A. S. et al. (2018)) que apresenta como tema os principais efeitos colaterais dos medicamentos emagrecedores e os riscos que eles trazem à saúde. A identificação dos artigos foi feita por meio de buscas em bases científicas, como Scientific Electronic Library Online (SciELO), PubMed, Google Scholar, Caribe 
em Ciências da Saúde (Lilacs) e Portal do Capes, selecionando os trabalhos publicados entre os anos de 2011 e 2021. Para a busca dos artigos, foram utilizados os termos chaves padronizados pelos Descritores em Ciências da Saúde (DeCS): Sibutramina, Orlistat, reações adversas, obesidade, corpo perfeito, medicamentos emagrecedores, risco, mídia, anorexígenos.

A seleção dos artigos baseou-se na conformidade dos limites dos assuntos aos objetivos deste trabalho. Como critérios de inclusão, foram considerados artigos em qualquer idioma e publicações realizadas nos últimos dez (10) anos. Sendo excluídos artigos que não incluíam essas exigências ou que não aborda os assuntos: reações adversas dos medicamentos anorexígenos e seus riscos à saúde.

\section{Resultados}

Dentre os 80.600 artigos encontrados através dos critérios selecionados para a realização do trabalho (publicações dos últimos 10 anos, buscados nas plataformas Bireme, PubMed, ScieLO, Portal do Capes, Lilacs e Google Scholar) foram selecionados apenas 14 trabalhos para a análise. Esses trabalhos selecionados abordam sobre os fatores de riscos ocasionados pelos medicamentos emagrecedores. A tabela 1 apresenta as principais características dos estudos selecionados (Tabela 1). 
Tabela 1. Distribuição das referências bibliográficas obtidas nas bases de dados Bireme, Google Scholar, Lilacs, SciELO, PubMed e Portal do CAPES de acordo com as palavras-chaves selecionadas.

\begin{tabular}{|c|c|c|c|c|c|}
\hline $\begin{array}{c}\text { Bases de } \\
\text { Dados }\end{array}$ & Estratégia de Busca & $\begin{array}{l}\text { Número de } \\
\text { Referências } \\
\text { Obtidas }\end{array}$ & $\begin{array}{c}\text { Resumos } \\
\text { Analisados }\end{array}$ & $\begin{array}{c}\text { Referências } \\
\text { Selecionadas para } \\
\text { Análise }\end{array}$ & $\begin{array}{l}\text { Selecionados } \\
\text { para Revisão }\end{array}$ \\
\hline \multirow[t]{5}{*}{ Google Scholar } & Obesidade & 40.400 & 18 & 12 & 2 \\
\hline & Corpo perfeito & 26.700 & 4 & 3 & 1 \\
\hline & Medicamentos para emagrecer & 2.490 & 6 & 2 & 1 \\
\hline & Sibutramina & 3.700 & 4 & 2 & 1 \\
\hline & Anorexígenos & 4.680 & 6 & 4 & 1 \\
\hline \multirow[t]{3}{*}{ BIREME } & Sibutramina & 13 & 13 & 6 & 0 \\
\hline & Orlistat & 1765 & 8 & 4 & 0 \\
\hline & Reações adversas orlistat & 6 & 5 & 3 & 0 \\
\hline PubMed & Anorectcsideeffect & 216 & 11 & 8 & 1 \\
\hline \multirow[t]{4}{*}{ Scielo } & Sibutramina & 40 & 7 & 3 & 1 \\
\hline & Anorexígenos & 24 & 10 & 5 & 2 \\
\hline & Sideeffect sibutramina & 2 & 2 & 2 & 1 \\
\hline & Liraglutida & 11 & 8 & 5 & 2 \\
\hline Portal do CAPES & Anorexígenos & 76 & 6 & 4 & 1 \\
\hline \multirow[t]{2}{*}{ LILACS } & Fármacos para obesidade & 339 & 4 & 3 & 1 \\
\hline & Anorexígenos & 138 & 3 & 3 & 1 \\
\hline
\end{tabular}

Fonte: Autores (2021).

Os artigos selecionados estão demonstrados na Tabela 2. Foram destacados os autores, o ano de publicação, a metodologia utilizada, os objetivos e os principais achados dos artigos que se enquadraram ao tema da presente revisão. 
Tabela 2. Distribuição das referências incluídas na revisão de literatura, de acordo com o autor e o ano de publicação, o tipo de estudo, os objetivos e os principais achados.

\begin{tabular}{|c|c|c|c|c|}
\hline $\mathbf{N}^{\mathbf{o}}$ & Autores (Ano) & Tipo de Estudo & Objetivos & Principais Achados \\
\hline 1 & $\begin{array}{l}\text { LONDOÑO-LEMOS, } \\
\text { Milton Enrique. } \\
\quad(2012)\end{array}$ & $\begin{array}{l}\text { Revisão detalhada, } \\
\text { sistemática. }\end{array}$ & $\begin{array}{l}\text { Esse estudo busca analisar a realidade do tratamento } \\
\text { para obesidade. Apesar de ter surgido muitos } \\
\text { medicamentos eficientes no mercado, o uso desses } \\
\text { fármacos têm representado sérias preocupações } \\
\text { quanto à segurança de seu uso, observando inúmeros } \\
\text { efeitos colaterais. }\end{array}$ & $\begin{array}{l}\text { O conhecimento sobre o eixo intestino-cérebro ajuda a entender como } \\
\text { funciona o sistema gastroenteropancreático, em relação a uma série de } \\
\text { peptídeos e hormônios que participam da regulação alimentar e do cérebro, } \\
\text { especificamente o hipotálamo como centro homeostático dos estados de } \\
\text { fome e anorexia, ou de apetite e, portanto, do peso corporal. Conhecendo } \\
\text { melhor o mecanismo de ação, as indicações de uso passam a ser mais } \\
\text { planejadas e com isso reduz os riscos de reações adversas. }\end{array}$ \\
\hline 2 & $\begin{array}{l}\text { FRANCO, Ruth } \\
\text { Rocha et al. (2014) }\end{array}$ & $\begin{array}{l}\text { Estudo duplo-cego } \\
\text { placebo controlado }\end{array}$ & $\begin{array}{l}\text { Avaliar o efeito da sibutramina na perda de peso de } \\
\text { adolescentes obesos. }\end{array}$ & $\begin{array}{l}\text { A sibutramina induziu significativamente mais perda de peso em } \\
\text { adolescentes obesos em comparação ao placebo, sem efeitos colaterais } \\
\text { significativos. A curva de evolução ponderal foi diferente de acordo com o } \\
\text { momento em que a sibutramina foi introduzida. Esse achado indica que o } \\
\text { melhor momento de introdução da sibutramina é quando a adesão começa a } \\
\text { falhar. }\end{array}$ \\
\hline 3 & $\begin{array}{c}\text { MOTA, Daniel } \\
\text { Marques et al. (2014) }\end{array}$ & $\begin{array}{l}\text { Estudo fármaco } \\
\text { econométrico }\end{array}$ & $\begin{array}{l}\text { Este estudo busca analisar os determinantes do } \\
\text { consumo de inibidores de apetite (anfepramona, } \\
\text { femproporex, mazindol e sibutramina) por meio da } \\
\text { estimação de um modelo dinâmico de dados em } \\
\text { painel para as capitais brasileiras e do Distrito } \\
\text { Federal (DF) no período de } 2009 \text { a } 2011 \text {. Avaliando } \\
\text { os riscos e problemas no uso dos mesmos.. }\end{array}$ & $\begin{array}{l}\text { A análise fármaco econométrica sugere que há problemas no uso racional } \\
\text { dos inibidores de apetite nas capitais brasileiras e no DF, seja no que tange } \\
\text { ao consumo desses medicamentos com outros fármacos - considerados } \\
\text { ilegais pelo Conselho Federal de Medicina e pela Anvisa - e, também, na } \\
\text { indicação terapêutica de uso desses produtos. }\end{array}$ \\
\hline 4 & $\begin{array}{l}\text { PAUMGARTTEN, } \\
\text { Francisco José Roma. } \\
\qquad(2017)\end{array}$ & Revisão sistemática & $\begin{array}{l}\text { Este estudo busca avaliar os riscos que as atuais } \\
\text { condutas médicas podem provocar na saúde dos } \\
\text { pacientes após a liberação da prescrição de fármacos } \\
\text { anorexígenos semelhantes à anfetamina no Brasil. }\end{array}$ & $\begin{array}{l}\text { O julgamento clínico é influenciado por crenças individuais, experiências } \\
\text { anteriores e educação e valores e, portanto, é passível de erros e vieses que } \\
\text { podem levar a decisões equivocadas sobre o tratamento mais eficaz e seguro } \\
\text { para o paciente. As anfetaminas podem levar a dependência além de } \\
\text { inúmeros efeitos adversos. }\end{array}$ \\
\hline
\end{tabular}




\begin{tabular}{|c|c|c|c|c|}
\hline 5 & $\begin{array}{l}\text { ZUBARAN, Carlos; } \\
\text { LAZZARETTI, } \\
\text { Rubia. (2013) }\end{array}$ & Estudo transversal & $\begin{array}{l}\text { Estabelecer a prevalência do uso de moderadores de } \\
\text { apetite entre estudantes da área da saúde na Região } \\
\text { Sul do Brasil. }\end{array}$ & $\begin{array}{l}\text { O uso durante a vida de moderadores de apetite foi significativo entre } \\
\text { estudantes da área da saúde, especialmente entre os matriculados em } \\
\text { Enfermagem e Nutrição. As drogas estimulantes simpatomiméticas foram os } \\
\text { agentes mais comumente usados. }\end{array}$ \\
\hline 6 & $\begin{array}{l}\text { ALARCÓN- } \\
\text { SOTELO, Alejandraet } \\
\text { al. (2018) }\end{array}$ & Revisão Sistemática & $\begin{array}{l}\text { Nesse artigo será realizada uma análise da eficácia } \\
\text { de cada um dos cinco medicamentos aprovados pelo } \\
\text { FDA hoje em dia para o tratamento de longo prazo } \\
\text { da obesidade: orlistat, fentermina / topiramato de } \\
\text { liberação prolongada, lorcaserina, naltrexona / } \\
\text { bupropiona liberação prolongada, e liraglutida. }\end{array}$ & $\begin{array}{l}\text { Resultados mostram que esses medicamentos se mostraram eficazes e com a } \\
\text { segurança necessária para serem considerados como opção terapêutica. }\end{array}$ \\
\hline 7 & $\begin{array}{l}\text { LÓPEZ-LÓPEZ, José } \\
\text { et al. (2016) }\end{array}$ & $\begin{array}{l}\text { Ensaio clínico } \\
\text { randomizado }\end{array}$ & $\begin{array}{l}\text { Revisar o efeito que os análogos do receptor do } \\
\text { GLP-1, incluindo a liraglutida, têm no controle dos } \\
\text { fatores de risco cardiovascular, particularmente } \\
\text { sobrepeso e obesidade, e na consequente prevenção } \\
\text { de doenças cardiovasculares. }\end{array}$ & $\begin{array}{l}\text { Os análogos do receptor do GLP-1 podem ser uma alternativa farmacológica } \\
\text { para o manejo do sobrepeso, obesidade e contribuir para a prevenção de } \\
\text { doenças cardiovasculares, embora ainda existam dúvidas sobre o benefício } \\
\text { de seu uso em nosso meio. }\end{array}$ \\
\hline 8 & $\begin{array}{l}\text { RADAELLI, Maqueli; } \\
\text { PEDROSO, Roberto } \\
\text { Costa; MEDEIROS, } \\
\text { Liciane Fernandes } \\
\text { (2016) }\end{array}$ & $\begin{array}{l}\text { Revisão } \\
\text { Bibliográfica }\end{array}$ & $\begin{array}{l}\text { Buscar informações sobre medicamentos } \\
\text { emagrecedores com o intuito de informar tanto os } \\
\text { benefícios quanto os riscos que esses medicamentos } \\
\text { trazem para a pessoa. }\end{array}$ & $\begin{array}{l}\text { Pode-se perceber que o tratamento com medicamentos é eficaz, porém o } \\
\text { tratamento somente com os fármacos não traz a cura para a obesidade. Se } \\
\text { faz necessário uma reeducação alimentar associadoa prática de exercícios } \\
\text { físicos. É de sumaimportância que um profissional especializado esteja } \\
\text { acompanhando o paciente durante o tratamento para o monitoramento dos } \\
\text { efeitos adversos. }\end{array}$ \\
\hline 9 & $\begin{array}{c}\text { DE ALENCAR, } \\
\text { Francisca Vitória } \\
\text { Rodrigues Silva; DOS } \\
\text { SANTOS } \\
\text { MEDEIROS, Cleyde; } \\
\text { BRITO, Maria Helena } \\
\text { Rodrigues Mesquita } \\
\text { (2019) }\end{array}$ & $\begin{array}{l}\text { Estudo exploratório, } \\
\text { descritivo } \\
\text { retrospectivo. }\end{array}$ & $\begin{array}{l}\text { O estudo tem o objetivo de mostrar quais os } \\
\text { medicamentos fitoterápicos mais utilizados para } \\
\text { emagrecer, assim como fazer uma avaliação sobre os } \\
\text { mesmos. }\end{array}$ & $\begin{array}{l}\text { Percebeu-se que os medicamentos fitoterápicos estavam sendo usados sem } \\
\text { uma orientação / prescrição médica ou farmacêutica. E que é necessário a } \\
\text { conscientização da população sobre os risco que o consumo indiscriminado } \\
\text { dos medicamentos fitoterápicos utilizados para emagrecer podem trazer para } \\
\text { a saúde. }\end{array}$ \\
\hline
\end{tabular}




\begin{tabular}{|c|c|c|c|}
\hline 10 & $\begin{array}{l}\text { SILVA, Natália } \\
\text { Cristina Sousa et al. } \\
\text { (2017) }\end{array}$ & Revisão Literária & $\begin{array}{l}\text { Buscou analisar fórmulas de medicamentos } \\
\text { emagrecedores de uma farmácia de manipulação e } \\
\text { quais danos eles podem causar nas } \\
\text { pessoas,principalmente os casos de pessoas obesas, } \\
\text { assim como seus benefícios, para mostrar opções de } \\
\text { uma melhor qualidade de vida. }\end{array}$ \\
\hline 11 & $\begin{array}{l}\text { VERRENGIA, } \\
\text { Elizabeth Cristina; } \\
\text { KINOSHITA, Samara } \\
\text { Alessandra Torquete; } \\
\text { AMADEI, Janete } \\
\text { Lane (2013) }\end{array}$ & Revisão Literária & $\begin{array}{l}\text { Fazer uma revisão da literatura sobre medicamentos } \\
\text { fitoterápicos que são usados no tratamento da } \\
\text { obesidade, a fim de informar sobre a eficiência, } \\
\text { segurança e toxicidades desses medicamentos. }\end{array}$ \\
\hline 12 & $\begin{array}{l}\text { SILVA, Eudiane dos } \\
\text { Santos et al. (2020) }\end{array}$ & Revisão Literária & $\begin{array}{l}\text { Averiguação dos efeitos farmacológicos da } \\
\text { Anfepramona e da Sibutramina, quando a ação } \\
\text { nutricional é falha. }\end{array}$ \\
\hline 13 & $\begin{array}{l}\text { ANDRADE, Tamires } \\
\text { Barreto et al. (2019) }\end{array}$ & $\begin{array}{l}\text { Revisão } \\
\text { Bibliográfica }\end{array}$ & $\begin{array}{l}\text { O artigo busca ampliar o conhecimento sobre os } \\
\text { riscos do uso irracional e exagerado do medicamento } \\
\text { emagrecedor Sibutramina. }\end{array}$ \\
\hline 14 & $\begin{array}{l}\text { NACCARATO, } \\
\text { Monique Campos; DE } \\
\text { OLIVEIRA LAGO, } \\
\text { Eloi Marcos (2014) }\end{array}$ & $\begin{array}{l}\text { Estudo descritivo, } \\
\text { retrospectivo de } \\
\text { revisão de literatura. }\end{array}$ & $\begin{array}{l}\text { Fazer uma comparação dos riscos e dos benefícios } \\
\text { que a Anfepramona e a Sibutramina podem trazer } \\
\text { para o organismo. }\end{array}$ \\
\hline
\end{tabular}

Fonte: Autores.
A mídia por padronizar um "corpo perfeito", influencia as pessoas a perder peso rapidamente através de medicamentos, porém esse consumo de medicamentos pode trazer consequências negativas para o organismo.

Muitos dos medicamentos fitoterápicos tem uma metodologia não confiável e toxicidades que não foram investigadas. Dos fitoterápicos analisados apenas um teve seu efeito confirmado.

O uso da Anfepramona e da Sibutramina nas dosagens adequadas e durante um período estabelecido tem efeitos positivos. Sendo necessários uma alimentação adequada assim como a prática de exercícios físicos. Assim os fármacos têm um papel auxiliar e não fundamental no tratamento contra a obesidade.

O uso da Sibutramina deve ser com cuidados, pois o mesmo apresenta efeitos colaterais assim como contra indicações, e pode causar dependência da pessoa ao medicamento. Logo, só deve ser usado nos casos em que o tratamento não-farmacológico não funcionar.

Tanto os riscos quanto os benefícios dos dois medicamentos dependem da dose, tolerância e do tempo de uso dos mesmos, se o paciente tem um tratamento não terapêutico em conjunto, e do histórico prévio do paciente em doenças psiquiátricas, hipertensão arterial, diabetes, dislipidemia, entre outros. 
Dos 14 artigos selecionados para a revisão, 3 Revisões Literárias; 2 Revisões Bibliográficas; 3 Revisões sistemáticas; 1 Estudo Duplo-Cego; 1 Estudo Fármaco Econométrico; 1 Estudo Transversal; 1 Ensaio Clínico Randomizado; 2 Estudos Descritivos, Retrospectivos.

\section{Discussão}

O tratamento para obesidade na grande maioria se faz pelo uso offlabel, ou seja, é o uso de drogas farmacêuticas que foram criadas para outros fins, mas que de certa forma tiveram bons resultados na perda de peso. Isso reflete de forma positiva na farmacologia, porém não afasta os efeitos adversos, inclusive, esses medicamentos merecem maior atenção, visto que não foram criados para o fim ao qual foi homologado, proporcionando maiores riscos de surgimento de efeitos adversos não estudados ou esperados pelos criadores da medicação. Portanto, o uso deve ser sempre orientado e físcalizado por um profissional (Silva B.L. \& Cantisani J.R., 2018; Paim M.B. \& Kovaleski D.F., 2020).

Entre vários fármacos offlabel usados na obesidade, um dos principais é a Sibutramina. A princípio foi criada para tratar a depressão, porém foi constatada a ineficiência para tal doença e a eficácia na perda de peso. Entretanto, notou-se que o uso excessivo pode implicar inúmeros riscos à saúde, como por exemplo, resistência bacteriana, hemorragia cerebral, taquicardia, ansiedade, convulsões, pressão alta, fadiga, constipação, taquicardia, anorexia, insônia, entre outros. Além disso, é capaz de agravar arritmias cardíacas, surtos psicóticos, elevação de pressão sanguínea, força de contração do miocárdio e provocar dependência química (Andrade T.B. et al., 2019; Silva N.C.S. et al., 2017).

O estudo Sibutramine Cardiovascular Outcomes (SCOUT), relata que o uso de Sibutramina em pacientes com mais de 55 anos, com doença cardiovascular e diabetes tipo 2, provoca um aumento do risco de eventos cardiovasculares em 16\% no grupo que fez uso dessa medicação.

Para Franco R.R. et al. (2014) as reações adversas provocadas pelo uso da Sibutramina de maior prevalência são: constipação, boca seca, alteração de humor, insônia, cefaleia e constipação. Já segundo Silva E.S. et al. (2020), a sibutramina pode causar como risco a saúde, taquicardia, elevação da pressão arterial, insônia, entre outros.

A sibutramina é um medicamento muito utilizado pelas pessoas para perder peso, porém ele causa alguns riscos à saúde, entre muitos deles, os mais preocupantes e mais frequentes são a insônia, elevação de pressão, taquicardia, cefaléia, ansiedade, irritabilidade (Radaelli M. et al., 2016).

Para Londoño-Lemos M.E. (2012), análogos de Anfetamina possuem alto potencial de causar dependência física e psicológica, podendo causar taquicardia, hipertensão, insônia e inquietação devido suas propriedades liberadoras de catecolaminas. A Tesofensina é um inibidor da recaptação de serotonina-noradrenalina-dopamina, e leva a efeitos adversos como o aumento da pressão arterial sistólica, sensação de boca seca, além de náusea, dor abdominal, constipação e diarreia.

Segundo Paumgartten F.J.R. (2017) o uso contínuo e a longo prazo de anorexígenos similares às anfetaminas podem causar dependência e casos de abuso. Esses medicamentos causam um aumento na frequência cardíaca e na pressão arterial devido seus efeitos adrenérgicos. Além disso, as anfetaminas são drogas psicoestimulantes e podem induzir insônia, alterações de humor e transtornos psiquiátricos. Dito isso, o uso dessa classe é desfavorável visto que os efeitos colaterais e o aumento da morbimortalidade cardíaca, se sobrepõem aos benefícios do medicamento. A sibutramina pode levar a quadros de constipação, aumento da pressão arterial e frequência cardíaca, boca seca e dor de cabeça. Diante desses efeitos adversos, seu uso deve ser evitado em pacientes com doenças cardiovasculares de alto risco.

Já segundo Mota D.M. et al. (2014). A combinação do medicamento fenfluramina e dexfenfluramina com fentermina ("fen-phen"), usados para tratamento de obesidade, quando usados por períodos mais longos podem causar valvulopatia cardíaca. Por esse motivo, chegou a ser retirada de mercado nos EUA. 
Para Naccarato M.C. \& De Oliveira Lago E.M. (2014), a sibutramina pode aumentar o risco de a pessoa ter um infarto do miocárdio não-fatal e de acidente vascular cerebral (AVC) não-fatal.

Outra forma de tratamento contra obesidade é a utilização do orlistat, porém ele pode causar uma série de efeitos adversos que muitas vezes atrapalham o paciente de dar continuidade ao tratamento. Os efeitos mais comuns estão relacionados ao sistema gastrointestinal, que incluem manchas oleosas, fezes líquidas, flatulência, dor abdominal e incontinência fecal. Por atuar no mecanismo de absorção de gorduras, o orlistat reduz a absorção de vitaminas lipossolúveis (A, D, E) sendo recomendado a esses pacientes a realização de suplementação. Além disso, foi relacionado também possíveis danos ao fígado pelo uso de orlistat (Londoño-Lemos M.E., 2012).

Já segundo Silva N.C.S. et al. (2017), o orlistat, outro medicamento utilizado para emagrecer, pode causar vários riscos, mas o principal é a redução na absorção de vitaminas lipossolúveis. Ainda segundo o autor outros riscos deste medicamento emagrecedor é o agravamento de vários problemas que a pessoa já possui como, por exemplo, de arritmias cardíacas, surtos psicóticos, elevação de pressão sanguínea, força de contração do miocárdio, e pode levar a pessoa a ter dependência química do medicamento

Radaelli M. et al. (2016) em seu artigo fala que praticamente todos os riscos que o orlistate traz à saúde da pessoa, são gastrointestinais por causa do efeito do medicamento, como por exemplo, fezes oleosas, flatulência, urgência fecal, entre outros.

Já segundo Alarcón-Sotelo A.et al. (2018) o tratamento farmacológico anti-obesidade possui seus efeitos adversos, que variam conforme o medicamento usado. No caso do Orlistat os sintomas estão relacionados ao sistema gastrointestinal, incluindo flatulência, esteatorreia, evacuação oleosa, urgência e incontinência fecal, dor abdominal, insuficiência hepática.

Inicialmente a anfepramona foi criada para tratar a narcolepsia e para o controle de crianças hipercinéticas, porém por apresentar um efeito que ajuda a diminuir a fome passou a ser mais utilizado como fármaco anorexígeno. Porém como efeito adverso que estão relacionados à ação noradrenérgica do medicamento, podendo causar boca seca, insônia, cefaleia, obstrução intestinal, irritabilidade entre outros, que são riscos à saúde (Silva E.S. et al., 2020).

Já segundo Radaelli M. et al. (2016), a anfepramona pode causar como risco para a saúde intoxicação aguda, alucinações, delírios, quadro psicótico, taquicardia, náusea, vômito, constipação intestinal, diminuição da libido.

Os fitoterápicos são outra fórmula farmacêutica muito usada com o intuito de perder peso, segundo De Alencar F.V.R.S. et al. (2019), o hibisco e a "seca barriga" são dois que foram muito usados entre 2017 e 2018 e mesmo com seus benefícios a saúde, eles podem causar risco como, por exemplo, tontura, enjoo, escurecimento da visão, sensação de fraqueza e desmaios no caso do hibisco; e mal-estar, ânsia de vômito, flatulência em excesso, desconforto abdominal e diarreia no caso do "seca barriga".

Segundo Verrengia E.C. et al. (2013), em seu artigo chamado Medicamentos Fitoterápicos no Tratamento da Obesidade, listaram alguns fitoterápicos usados para emagrecer, sendo os três que se destacam são o Citrusauratium, Phaseolusvulgaris e Camelliasinesis, alguns dos risco que esses fitoterápicos trazem a saúde são aumento de pressão arterial e frequência cardíaca (Citrusauratium); ansiedade e taquicardia (Camelliasinesis); hipertrofia do pâncreas, hipersecreção de enzimas digestivas e má absorção intestinal (Phaseolusvulgaris).

Segundo Radaelli M. et al. (2016), a GarciniaCambogia, uma planta nativa do sul da Índia, muito usada como tempero, porém atualmente usado para controlar e/ou reduzir peso, pode trazer risco à saúde por causar efeitos adversos como hepatotoxicidade, infecção de garganta, pneumonia e hemorragia.

Para López-López J. et al. (2016) a presença de efeitos adversos graves, como pancreatite aguda e câncer com o uso de liraglutida e exenatida podem ser descartados, pois não foi possível documentar nenhuma associação dessas drogas com esses efeitos. Dessa forma, o autor conclui que não há evidências suficientes para considerar que esses eventos são 
desencadeados por incretinas.Tal estudo bate de frente com as recomendações de segurança para o uso de liraglutida da American FoodandDrugAdministration (FDA, FoodandDrugAdministration). Na qual relata o aumento do risco de desenvolver pancreatite devido ao mecanismo de ação do GLP-1.

Segundo Alarcón-Sotelo A. et al. (2018), o tratamento usando a Liraglutida, pode gerar como efeito adverso náusea, vômito, diarreia, prisão de ventre, hipoglicemia, colecistite e pancreatite, que podem trazer risco à saúde da pessoa.

A Lorcaserina é outro medicamento usado e causa cefaleia, tontura, sonolência, visão turva, náusea, boca seca, fadiga, ressecamento, nasofaringite. Fentermina / Topiramato, provocam sensação de boca seca, alterações do paladar, parestesia, tontura, insônia, palpitações, prisão de ventre, disgeusia. A Naltrexona / bupropiona leva a quadros de náusea, vômito, cefaleia, tontura, insônia, prisão de ventre, pressão arterial elevada (Alarcón-Sotelo A. et al. 2018).

\section{Considerações Finais}

É possível perceber que a sociedade atual é bastante influenciada pela mídia, principalmente o público mais jovem. Isso é um fator preocupante, pois a mídia tende a mascarar o lado real e negativo, além de fantasiar e criar tendências que beneficiam o mercado financeiro. Isso acontece no padrão de beleza e corpo ideal, influenciando a sociedade a banalizar os riscos para conseguir o padrão da magreza. Isso tem implicado no uso indiscriminado de anorexígenos, sem orientação e indicação.

Os riscos e efeitos adversos muitas vezes não são revelados aos pacientes, o que leva muitas pessoas, principalmente as do sexo feminino, acreditarem na fórmula mágica do emagrecimento rápido com uso de medicamentos como uma solução benéfica. Portanto, já é sabido que obesidade é uma doença, logo, assim como qualquer outra tem portadores da mesma e esses sim devem receber o tratamento, quando indicado, avaliado e prescrito por um profissional capacitado da área.

Portando, este presente estudo surge com a ideia de apresentar o lado obscuro do uso indiscriminado de fármacos anorexígenos, relatando vários efeitos colaterais que todo paciente ao fazer a utilização dos medicamentos está sujeito a possuir. E, além disso, atentar a todos a necessidade de orientação de uso e de maior fiscalização diante da liberação dos anorexígenos. Salienta-se a necessidade de ser estudado mais detalhadamente em outras populações, visando resultados adicionais que possam vir a colaborar com nossos achados.

\section{Referências}

ABESO, (2016). Diretrizes brasileiras de obesidade: 2016. Associação Brasileira para Estudo da Obesidade e da Síndrome Metabólica (ABESO). São Paulo.

Alarcón-Sotelo, A. et al. (2018).Actualidadeseneltratamiento farmacológico a largo plazo de laobesidad.¿ Una opciónterapéutica?. Medicina interna de México. 34(6), 946-958.

Andrade, T.B. et al.(2019). O Farmacêutico Frente aos Riscos do Uso de Inibidores de Apetite: A Sibutramina. Revista Científica da Faculdade de Educação e Meio Ambiente, 10(1),81-92.

De Alencar, F.V.R.S. et al.(2019). O uso de medicamentos fitoterápicos como emagrecedores em uma cidade do Maranhão.Research, Society and Development, 9(2),e135922096.

De Oliveira A.V. et al. (2016) IInfluência da mídia na construção da autoimagem de jovens na sociedade pós-moderna: A busca do corpo perfeito. Revista Científica da FEPI-Revista Científic@ Universitas.

De Menezes T.S.B. et al. (2021) Representação Social da obesidade: análise com estudantes do ensino médio e universitários. Ciencias Psicológicas.

Dias P.C. et al. (2017) Obesidade e políticas públicas: concepções e estratégias adotadas pelo governo brasileiro. Cadernos de Saúde Pública, 33 , e00006016.

Franco R.R. et al. (2014) The effect of sibutramine on weight loss in obese adolescents. Arquivos brasileiros de endocrinologia e metabologia.

Lima A.F. et al. (2013). A ideologia do corpo feminino perfeito: questões com o real. Psicologia em Estudo, 18(1),49-59.

Londoño-Lemos M.E. (2012). Tratamiento farmacológico contra laobesidad. Revista Colombiana de Ciencias Químico-Farmacéuticas, 41(2), $217-261$. 
Research, Society and Development, v. 10, n. 10, e535101019147, 2021

(CC BY 4.0) | ISSN 2525-3409 | DOI: http://dx.doi.org/10.33448/rsd-v10i10.19147

López-López J.et al. (2016). ¿ Existe unespacio para los análogos de laincretina como terapia para el sobrepeso, laobesidad y laprevención de laenfermedadcardio-metabólica?. Revista Colombiana de Cardiología, 23(3), 200-209.

Mota D.M. et al. (2014).Are there irrationalities in the consumption of anti-obesity drugsin Brazil? apharmaco-econometric analysis of panel datasets.Ciencia\&saude coletiva, 19(5), 1389-1400.

Naccarato M.C. \& De Oliveira Lago E.M. (2014). Uso dos anorexígenos anfepramona e sibutramina: Benefício ou prejuízo à saúde?.Revista Saúde-UNG-Ser, $8(1-2), 66-72$

Paim M.B. \& Kovaleski D.F. (2020).Análise das diretrizes brasileiras de obesidade: patologização do corpo gordo, abordagem focada na perda de peso e gordofobia.Saúde e Sociedade, 29, e190227.

Paumgartten F.J.R. (2017). The return of amphetamine-like anorectics: a backward step in the practice of evidence-based medicine in Brazil.Cadernos de saudepublica, 33, e00124817.

Radaelli M. et al. (2016). Farmacoterapia da Obesidade: Benefícios e Riscos. Saúde e Desenvolvimento Humano, 4(1), 101-115.

Lucema B.B. et al.. (2020). Ninguém é tão perfeito que não precise ser editado: fetiche e busca do corpo ideal. Psicologia USP, 31 .

Silva B.L. \& Cantisani J.R. (2018). Interfaces entre a gordofobia e a formação acadêmica em nutrição: um debate necessário. DEMETRA: Alimentação, Nutrição \& Saúde, 13(2), 363-380.

Silva E.S.et al. (2020). O Uso Farmacológico De Anfepramona e Sibutramina no Tratamento Coadjuvante da Obesidade. Congresso Brasileiro de Ciência da Saúde.

Silca N.C.S.et al. (2017). Análise da utilização de medicamentos emagrecedores dispensados em farmácias de manipulação de Ipatinga-MG.Única Cadernos Acadêmicas, 3(1).

Tarozo M. \& Pessa R.P. (2020). Impacto das consequências psicossociais do estigma do peso no tratamento da obesidade: uma revisão integrativa da literatura.Psicologia: Ciência e Profissão, 40.

Tylka T.L. et al. (2014). The weight-inclusive versus weight-normative approach to health: Evaluating the evidence for prioritizing well-being over weight loss.Journalofobesity, 2014.

Verrengia E.C. et al. (2013). Medicamentos Fitoterápicos no tratamento da obesidade.Uniciência, 17(1).

Zubaran C. \& Lazzaretti R. (2013). Uso de moderadores de apetite entre estudantes da área da saúde na Região Sul do Brasil. Einstein (São Paulo)11(1), 4752 . 